\title{
Axillary lymphadenitis due to Mycobacterium avium-intracellulare
}

\author{
E. W. COLLEY \\ F.R.C.Path. \\ ROSAMOND A. COX \\ B.M., B.Ch.
}

Department of Pathology, Kettering General Hospital, Rothwell Road, Kettering, Northants NN16 8UZ

\begin{abstract}
Summary
A 5-year-old girl presented with a swelling in the right axilla from which Mycobacterium avium-intracellulare was cultured. The gland was excised and she made an uneventful recovery.
\end{abstract}

KEY WORDS: lymphadenitis, Mycobacterium avium-intracellulare.

\section{Introduction}

The avium-intracellulare group of Mycobacteria is a well-recognised cause of cervical lymph node infection in children. Nodes at other sites, such as the submandibular region and the groin, are less commonly involved. In an intensive search of the literature, Lincoln and Gilbert (1972) found only 9 cases of axillary lymph node disease in children which were caused by non-tuberculous mycobacteria.

\section{Case report}

A 5-year-old girl presented with a 3-day history of a painful swelling in the right axilla. Examination revealed a large mass of matted lymph nodes measuring $4 \times 3 \mathrm{~cm}$. No other nodes were involved and she was otherwise in good health. Investigations showed a haemoglobin concentration of $12.9 \mathrm{~g} / \mathrm{dl}$, white cell count $6.7 \times 10^{9} /$ litre, erythrocyte sedimentation rate $7 \mathrm{~mm}$ in first hour. Chest X-ray was normal. A provisional diagnosis of staphylococcal lymphadenitis was made and she was treated with cloxacillin. One week later, the size of the mass was unchanged and a discharging sinus was now present. A Mantoux test was strongly positive at 1:10000 dilution (equivalent to one unit of tuberculin). The mass was biopsied and histology showed caseating tuberculous granulation tissue with numerous epithelioid cells, Langhans' giant cells and lymphocytes. Ziehl-Neelsen and auramine staining for acid-fast bacilli were negative, but culture yielded a Mycobacterium of the avium-intracellulare group, sensitive only to ethionamide and cycloserine. Anti-tuberculous therapy was begun whilst the results of culture were awaited and the patient was started on isoniazid, $150 \mathrm{mg}$ b.d., and rifampicin, $200 \mathrm{mg}$ b.d. Tịis was discontinued after only 4 days when the patie was readmitted with severe vomiting and found to have abnormal liver function tests.

After 7 weeks the tests returned to normal and tife entire axillary mass was then excised. Two months later, the patient remained well, the wound was fuly healed and there was no evidence of further lymph node enlargement.

\section{Discussion}

Mycobacterium avium-intracellulare causes 220 distinct forms of disease in man. Most cases in a fupfs occur as pulmonary disease, usually affecting middle-aged men with pre-existing lung damage. In young children, it most commonly causes infection of the cervical lymph nodes. Clinically, lymphadeniक due to non-tuberculous mycobacteria differs frofn that caused by M.tuberculosis. It has been suggested by Lincoln and Gilbert (1972) and Wolinsky (19/ק) that the following features indicate infection with acid-fast bacilli other than M.tuberculosis: (1) ade tis in a child 1-4-years-old; (2) no history of contaet with a tuberculous person; (3) non-reactive tubercislin tests in siblings; (4) normal chest X-ray; (5) failurie of appreciable response to anti-tuberculous drugs; early suppuration; (7) unilateral lymph node enlarge ment. The patient described here was aged 5 yea Her 8-year-old sub-normal brother was not tuberculin tested. The unacceptable side effects caused by the drugs meant that response to chemotherapy coutd not be assessed. In other respects, her disease fulfills the above criteria.

N

Diagnosis depends upon culturing the organism from the affected gland. Differential skin testig using antigens prepared from the avium intracellux lare group and PPD is often helpful but cress reactions may occur (Wolinsky, 1979; Chapmag, 1982).

It is important to distinguish infection due to M.avium-intracellulare from tuberculosis because the 
two diseases present fundamental differences in their epidemiology and management. M.avium-intracellulare is resistant to most anti-tuberculous drugs and the treatment of choice is excision of the gland.

M.avium-intracellulare is widely distributed in the environment, in soil, house dust, water and in domestic animals. Meissner and Anz (1977) pointed out that chickens and wild birds are the natural hosts of certain types of M.avium and suggested that they were an important source of human infection. Human infection is thought to result from inhalation, ingestion or direct inoculation of the skin through minor abrasions. As the organism is derived from the environment, tracing and surveillance of contacts is unnecessary.

\section{Acknowledgments}

We thank Dr E. H. Smith, Consultant Paediatrician, for allowing us to report this case of a patient under his care, and Dr P. A. Jenkins, Director of the Mycobacterium Reference Unit, Public Health Laboratory, Cardiff, for his advice, and for identification and culture of the organism.

\section{References}

Chapman, J.S. (1982) The atypical mycobacteria. American Review of Respiratory Diseases, 125, 119.

LINCOLN, E.M. \& GILBERT, L.A. (1972) Diseases in children due to Mycobacteria other than Mycobacterium tuberculosis. American Review of Respiratory Diseases, 105, 683.

MEISSNER, G. \& ANZ, W. (1977) Sources of Mycobacterium avium complex infection resulting in human diseases. American Review of Respiratory Diseases, 116, 1057.

WoLINSKY, E. (1979) Non-tuberculous mycobacteria and associated diseases. American Review of Respiratory Diseases, 119, 107.

(Accepted 5 January 1983) 